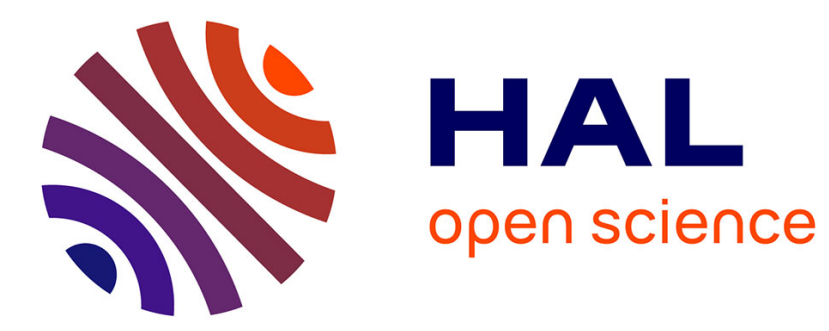

\title{
The geomagnetic secular-variation timescale in observations and numerical dynamo models
}

Florian Lhuillier, Alexandre Fournier, Gauthier Hulot, Julien Aubert

\section{To cite this version:}

Florian Lhuillier, Alexandre Fournier, Gauthier Hulot, Julien Aubert. The geomagnetic secularvariation timescale in observations and numerical dynamo models. Geophysical Research Letters, 2011, 38 (9), pp.L09306. 10.1029/2011GL047356 . insu-01410184

\section{HAL Id: insu-01410184 \\ https://hal-insu.archives-ouvertes.fr/insu-01410184}

Submitted on 6 Dec 2016

HAL is a multi-disciplinary open access archive for the deposit and dissemination of scientific research documents, whether they are published or not. The documents may come from teaching and research institutions in France or abroad, or from public or private research centers.
L'archive ouverte pluridisciplinaire HAL, est destinée au dépôt et à la diffusion de documents scientifiques de niveau recherche, publiés ou non, émanant des établissements d'enseignement et de recherche français ou étrangers, des laboratoires publics ou privés. 


\title{
The geomagnetic secular-variation timescale in observations and numerical dynamo models
}

\author{
Florian Lhuillier, ${ }^{1}$ Alexandre Fournier, ${ }^{1}$ Gauthier Hulot, ${ }^{1}$ and Julien Aubert ${ }^{1}$
}

Received 3 March 2011; accepted 29 March 2011; published 5 May 2011.

[1] The knowledge of the spatial power spectra of the main geomagnetic field and of its secular variation makes it possible to define typical timescales $\tau_{n}$ for each spherical harmonic degree $n$. Investigating both observations and numerical dynamos, we show that a one-parameter law of the form $\tau_{n}=\tau_{\mathrm{SV}} / n$ is satisfied for the non-dipole field, given the statistical way the observed $\tau_{n}$ are expected to fluctuate. Consequently, we determine the corresponding secularvariation timescale $\tau_{\mathrm{SV}}$ from either instantaneous or timeaveraged spectra, leading to a value of $415 \pm_{45}^{55} \mathrm{yr}$ for recent satellite field models. In the broader context of geomagnetic data assimilation, $\tau_{\mathrm{SV}}$ could provide a sensible and convenient means to rescale the time axis of dynamo simulations. Citation: Lhuillier, F., A. Fournier, G. Hulot, and J. Aubert (2011), The geomagnetic secular-variation timescale in observations and numerical dynamo models, Geophys. Res. Lett., 38, L09306, doi:10.1029/2011GL047356.

\section{Introduction}

[2] Considering the main magnetic field at the Earth's surface, it is possible to define the mean square field due to all spherical harmonic terms of degree $n$ [Mauersberger, 1956; Lowes, 1966],

$$
R_{n}=(n+1) \sum_{m=0}^{n}\left[\left(g_{n}^{m}\right)^{2}+\left(h_{n}^{m}\right)^{2}\right],
$$

where $\left\{g_{n}^{m}, h_{n}^{m}\right\}$ are the Gauss coefficients of spherical harmonic degree $n$ and order $m$ normalised according to the convention of Schmidt.

[3] Analogous quantities can also be defined for the rate of change of the field [Lowes, 1974],

$$
Q_{n}=(n+1) \sum_{m=0}^{n}\left[\left(\dot{g}_{n}^{m}\right)^{2}+\left(\dot{h}_{n}^{m}\right)^{2}\right],
$$

where $\left\{\dot{g}_{n}^{m}, \dot{h}_{n}^{m}\right\}$ are the time derivatives of the Gauss coefficients. The graphical representations of $R_{n}$ and $Q_{n}$ as a function of $n$ are known as the spatial power spectra of the field and of its secular variation.

[4] For each spherical harmonic degree $n$, it is then possible to define the following timescales [Stacey, 1992, p. 355],

$$
\tau_{n}=\sqrt{\frac{R_{n}}{Q_{n}}}=\sqrt{\frac{\sum_{m=0}^{n}\left[\left(g_{n}^{m}\right)^{2}+\left(h_{n}^{m}\right)^{2}\right]}{\sum_{m=0}^{n}\left[\left(\dot{g}_{n}^{m}\right)^{2}+\left(\dot{h}_{n}^{m}\right)^{2}\right]}},
$$

\footnotetext{
${ }^{1}$ Institut de Physique du Globe de Paris, Sorbonne Paris Cité, Université Paris Diderot, INSU/CNRS, UMR 7154, Paris, France.

Copyright 2011 by the American Geophysical Union. 0094-8276/11/2011GL047356
}

which characterise the dynamics of the main field, independently of the distance from which it is observed. They are defined here in terms of instantaneous values, but a definition based on averaged values,

$$
\tau_{n}=\sqrt{\frac{\left\langle R_{n}\right\rangle}{\left\langle Q_{n}\right\rangle}}
$$

where the brackets \langle\rangle denote time averaging, has also been used [Christensen and Tilgner, 2004]. Several ways of interpreting these timescales have been proposed. In particular they can be interpreted as reorganisation times [Stacey, 1992], in which case they are given a phenomenological interpretation. They can also be interpreted as correlation times [Hulot and Le Mouël, 1994], in which case they are given a statistical interpretation. They measure how long it would take for the field at spherical harmonic degree $n$ to be completely renewed.

[5] These timescales are known to decrease with increasing spherical harmonic degree $n$, but the exact dependence of $\tau_{n}$ with respect to $n$ is still a matter of debate. A twoparameter law of the form $\tau_{n}=\delta \times n^{-\gamma}$ has on the one hand been assumed for recent satellite data [Holme and Olsen, 2006; Olsen et al., 2006; Lesur et al., 2008; Hulot et al., 2010a], where the least-squares estimate of the exponent $\gamma$ varies between 1.32 and 1.45 , depending on the epoch and the range of spherical harmonic degrees considered for the fit. A simpler one-parameter law of the form $\tau_{n}=\tau_{\mathrm{SV}} / n$ has on the other hand been proposed for dynamo solutions [Christensen and Tilgner, 2004], where $\tau_{\mathrm{SV}}$ is the so-called secular-variation timescale.

[6] In this letter, we test the relevance of the simpler oneparameter law, investigating both observations and numerical dynamos. We explore to what extent such an inverse linear law is acceptable given the way the observed $\tau_{n}$ are expected to fluctuate, and discuss why this one-parameter law can be satisfactorily used in contrast to a more general two-parameter power law. We finally contemplate the possibility of using the secular-variation timescale as a means to rescale the time axis of dynamo simulations, in the prospect of comparing numerical predictions with data.

\section{Method}

[7] To address the above questions, one needs to resort to a statistical description of the geomagnetic field. One such description is provided by the stationary isotropic statistical (SIS) model of the geomagnetic field proposed by Hulot and Le Mouël [1994]. It assumes that the set $\left\{g_{n}^{m}, h_{n}^{m}\right\}$ consists of independent stationary Gaussian processes with zero mean and variance $\sigma_{n}^{2}$. Under this assumption, the time derivatives 
$\left\{\dot{g}_{n}^{m}, \dot{h}_{n}^{m}\right\}$ are also a set of independent stationary Gaussian processes with zero mean and some variance $\sigma_{n}^{\prime 2}$. Within this statistical framework, the mathematical expectations of $R_{n}$ and $Q_{n}$ are respectively $\bar{R}_{n}=(n+1)(2 n+1) \sigma_{n}^{2}$ and $\bar{Q}_{n}=$ $(n+1)(2 n+1) \sigma_{n}^{\prime 2}$. As shown by Hulot and Le Mouël [1994], it entails that the quantities $X_{n}=(2 n+1) R_{n} / R_{n}$ and $Y_{n}=(2 n+1) Q_{n} / \bar{Q}_{n}$ both follow $\chi^{2}$-distributions with $(2 n+1)$ degrees of freedom, and that the ratio $X_{n} / Y_{n}$ follows a $F$-distribution with $(2 n+1,2 n+1)$ degrees of freedom [von Storch and Zwiers, 2001, section 2.7.10]. Another expression of the ratio $X_{n} / Y_{n}$ is $\left(\tau_{n} / \bar{\tau}_{n}\right)^{2}$, where $\bar{\tau}_{n}=\left(\bar{R}_{n} / \bar{Q}_{n}\right)^{1 / 2}$. It implies that $\tau_{n}$ is expected to fluctuate about $\bar{\tau}_{n}$ in such a way that $\left(\tau_{n} / \bar{\tau}_{n}\right)^{2}$ follows a $F$-distribution with $(2 n+1,2 n+1)$ degrees of freedom, whose probability density function (pdf) will be denoted hereinafter by $F^{2 n+1,2 n+1}$. When working on averaged quantities as defined in equation (4), this formalism must be modified to account for the increase in the number of degrees of freedom, since two contiguous values of a Gauss coefficient of degree $n$ are expected to become statistically independent after approximately $3 \cdot \bar{\tau}_{n}$ [Hongre et al., 1998]. If $T$ denotes the width of the temporal window over which the secular variation is studied, the number of independent Gauss coefficients defining $\left\langle R_{n}\right\rangle$ and $\left\langle Q_{n}\right\rangle$ is multiplied by $N_{n}=T /\left(3 \cdot \bar{\tau}_{n}\right)$ (rounded to the nearest larger integer), and the ratio $\left(\tau_{n} / \bar{\tau}_{n}\right)^{2}$ consequently follows a $F$-distribution with $\left((2 n+1) N_{n},(2 n+1) N_{n}\right)$ degrees of freedom.

[8] Let us now consider the two models we wish to test for $\bar{\tau}_{n}$, and the associated forward modelling relationships linking the parameter space $\mathcal{M}$ to the data space $\mathcal{O}=\left\{\tau_{n}^{\text {obs }}\right\}_{n=N_{0}}^{N}$, where the $\tau_{n}^{\text {obs }}$ are the correlation times observed from spherical harmonic degree $n=N_{0}$ to $N$. In the case of the one-parameter inverse linear law, $\mathcal{M}=\left\{\tau_{\mathrm{SV}}\right\}$ and the forward modelling relationship is $\bar{\tau}_{n}=\tau_{\mathrm{SV}} / n$. In the case of the two-parameter power law, $\mathcal{M}=\{\delta, \gamma\}$ and the forward modelling relationship is $\bar{\tau}_{n}=\delta \times n^{-\gamma}$. No prior information is assumed on either $\mathcal{M}$. According to the SIS model, observations consisting of instantaneous correlations times (based on instantaneous spectra, and defined in equation (3)) are expected to follow a distribution described by the probability

$$
g_{\text {obs }}\left(\left\{\tau_{n}^{\mathrm{obs}}\right\}_{n=N_{0}}^{N}\right)=\prod_{n=N_{0}}^{N} F^{2 n+1,2 n+1}\left[\left(\frac{\tau_{n}^{\mathrm{obs}}}{\bar{\tau}_{n}}\right)^{2}\right] .
$$

We now seek best estimates of either $\tau_{\mathrm{SV}}$ or $(\delta, \gamma)$ such that, for each degree $n$, the observation $\tau_{n}^{\text {obs }}$ is consistent with the $F^{2 n+1,2 n+1}$-distribution we expect $\left(\tau_{n}^{\text {obs }} / \bar{\tau}_{n}\right)^{2}$ to follow. Note that, since each pdf $F^{2 n+1,2 n+1}(x)$ is skewed (see Figure 2), the median $x=1$ differs from the likeliest value $x=\chi_{2 n+1}$, where $\chi_{K}=(K-2) /(K+2)$. Searching for the parameters, which characterise $\bar{\tau}_{n}$ by maximising the probability defined by equation (5), would thus lead to a biased estimator, achieving a best fit of $\bar{\tau}_{n}$ to $\tau_{n}^{\text {obs }} / \sqrt{\chi_{2 n+1}}$ rather than to $\tau_{n}^{\text {obs }}$. A rigorous treatment of this issue could involve procedures such as a uniformisation of the statistics [see, e.g., Khokhlov et al., 2001]. We however prefer to adopt a simpler approximate way to proceed, and rescale $\tau_{n}^{\text {obs }}$ by a factor $\sqrt{\chi_{2 n+1}}$ before seeking the parameters that maximise the correspondingly rescaled probability. The robustness of this procedure was checked using synthetic data (not shown). We accordingly resort to the probability

$$
f\left(\tau_{\mathrm{SV}}\right)=\frac{1}{\nu} \prod_{n=N_{0}}^{N} F^{2 n+1,2 n+1}\left[\chi_{2 n+1}\left(n \frac{\tau_{n}^{\mathrm{obs}}}{\tau_{\mathrm{SV}}}\right)^{2}\right]
$$

to estimate $\tau_{\mathrm{SV}}$ in the one-parameter case, and

$$
f(\delta, \gamma)=\frac{1}{\nu} \prod_{n=N_{0}}^{N} F^{2 n+1,2 n+1}\left[\chi_{2 n+1}\left(\frac{\tau_{n}^{\mathrm{obs}}}{\delta \times n^{-\gamma}}\right)^{2}\right]
$$

to estimate $\delta$ and $\gamma$ in the two-parameter case, where $\nu$ is a normalisation factor in both equations. If we consider "time-averaged" correlation times (based on time-averaged spectra, and defined in equation (4)) instead of instantaneous ones, the line of reasoning remains the same, provided that $F^{2 n+1,2 n+1}$ and $\chi_{2 n+1}$ are respectively changed to $F^{(2 n+1) N n,(2 n+1) N_{n}}$ and $\chi_{(2 n+1) N_{n}}$, for the reasons outlined above. Using equation (6), the maximum of likelihood of $\tau_{\mathrm{SV}}$, denoted by $\tau_{\mathrm{SV}}^{\max }$, can then be deduced along with its $90 \%$ confidence interval for both observations and numerical dynamos. In addition, equation (7) allows us to map the two-dimensional pdf of $(\delta, \gamma)$, thereby making it possible to assess the robustness of the inverse linear law against the more general two-parameter power law.

\section{Secular-Variation Timescale in Geomagnetic Field Models}

[9] In this section we consider historical and contemporary core field models. We restrict our attention to the "observatory" part of gufml [Jackson et al., 2000], to wit the period 1840-1990. We prefer not to use the model for earlier epochs because the correlation times for the higher degrees $(n \geq 5)$ are more heavily affected by the temporal regularisation. We also consider the short-term satellite models POMME-6 [Maus et al., 2010], GRIMM-2 [Lesur et al., 2010] and CHAOS-3 [Olsen et al., 2010], all of which contributed to the elaboration of the IGRF-11 [Finlay et al., 2010].

[10] We first consider the relevance of the one-parameter inverse linear law $\bar{\tau}_{n}=\tau_{\mathrm{SV}} / n$. Because of a large non-zero averaged value of the axial dipole $g_{1}^{0}$ [see, e.g., Hulot et al., 2010a], which violates the SIS assumption, we discard $\tau_{1}^{\text {obs }}$ and rely on values of $\tau_{n}^{\text {obs }}$ computed from equation (3) for $n=2-13$ (resp. from equation (4) for $n=2-10$ ) for the short-term satellite models (resp. for $g u f m 1$ ). The upper bound value of $n=13$ for recent satellite models was chosen because of the remarkable agreement of $Q_{n}$ for these recent field models up to that degree, and because crustal contamination makes the $n>13$ part of $R_{n}$ irrelevant for this study [see e.g., Hulot et al., 2010a]. Table 1 presents the recovered values of $\tau_{\mathrm{SV}}^{\max }$ with their $90 \%$ confidence interval, based on equation (6). Note the good agreement amongst the satellite models. The historical model gufml leads to different values of $\tau_{\mathrm{SV}}$, which are nevertheless consistent with the expected uncertainties.

[11] Figure 1 presents the observed correlation times $\tau_{n}^{\text {obs }}$ (black stars) with their corresponding fits (red lines) for CHAOS-3 (Figure 1a) and gufml (Figure 1b). To check the 
Table 1. Values of $\tau_{\mathrm{SV}}^{\max }$ With Their $90 \%$ Confidence Interval for Various Geomagnetic Field and Dynamo Models When Computing $\tau_{n}^{\text {obs }}$ Either From Equation (3) or From Equation (4) ${ }^{\mathrm{a}}$

\begin{tabular}{lcccc}
\hline \multicolumn{1}{c}{ Model } & Equation & $n$ & $\tau_{\mathrm{SV}}^{\max }$ & $\tau_{\mathrm{SV}}$ \\
\hline CHAOS-3 2005 & 3 & $2-13$ & {$[378 ; \mathbf{4 2 5} ; 483]$} & 445 \\
GRIMM-2 2005 & 3 & $2-13$ & {$[361 ; \mathbf{4 0 6} ; 461]$} & 446 \\
POMME-6 2005 & 3 & $2-13$ & {$[368 ; \mathbf{4 1 3} ; 470]$} & 443 \\
gufm1 1840-1990 & 4 & $2-10$ & {$[423 ; \mathbf{4 8 9} ; 579]$} & 388 \\
dynamo model & 3 & $2-13$ & {$[0.129 ; \mathbf{0 . 1 4 6} ; 0.164]$} & 0.136 \\
dynamo model & 4 & $2-13$ & {$[0.137 ; \mathbf{0 . 1 4 0} ; 0.143]$} & 0.140 \\
\hline
\end{tabular}

${ }^{\mathrm{a}}$ Values for the geomagnetic field models are expressed in years, whereas those for the dynamo model are in units of the viscous diffusion time. The last column indicates the values of $\tau_{\mathrm{SV}}$ recovered from a standard leastsquares algorithm. Values in bold are $\tau_{\mathrm{SV}}^{\max }$, and values in brackets are their $90 \%$ confidence interval.

compliance of the inferred law $\bar{\tau}_{n}=\tau_{\mathrm{SV}}^{\max } / n$ with the $\tau_{n}^{\text {obs }}$, Figure 1 also presents $90 \%$ statistical dispersion bars, deduced from the $F^{2 n+1,2 n+1}$-pdf for $\left(\tau_{n} / \bar{\tau}_{n}\right)^{2}$, in agreement with the SIS model. Note that for this a posteriori check, the exact (and not the rescaled) $F^{2 n+1,2 n+1}$ are used, as our goal is no longer to search for an optimal $\tau_{\mathrm{SV}}$, but instead to verify the compatibility of the observations with their expected statistics. Note also that in the case of $g u f m 1$, the factor $N_{n}$ introduced above is always equal to one, which led us to resort to $F^{2 n+1,2 n+1}$-pdfs, even though we computed "time-averaged" values of $\tau_{n}^{\text {obs }}$ using equation (4). We obtain that the observations (black stars) are all located within the $90 \%$ statistical dispersion bars, confirming the compatibility of the inverse linear law $\tau_{\mathrm{SV}}^{\max } / n$ with the observed geomagnetic field, within the statistical framework we used.

[12] Let us now consider the relevance of the more general two-parameter power law $\bar{\tau}_{n}=\delta \times n^{-\gamma}$. In order to assess which sets of values $(\delta, \gamma)$ can possibly provide a better fit to the data, we rely on equation (7) to compute $f(\delta, \gamma)$. Figures $1 \mathrm{e}$ and $1 \mathrm{f}$ show these pdfs (normalised by their maximum, and hereinafter denoted by $f$ ), obtained for CHAOS-3 and gufm 1, respectively. Figures $1 \mathrm{e}$ and $1 \mathrm{f}$ reveal crescent-shaped $f>0.8$ surfaces (in red) which intersect the $\gamma=1$ axis. Therefore, even if particular values of $(\delta, \gamma)$ can provide a better fit to the data than $(\delta, \gamma)=\left(\tau_{\mathrm{SV}}, 1\right)$, this improvement is marginal, and not statistically significant.
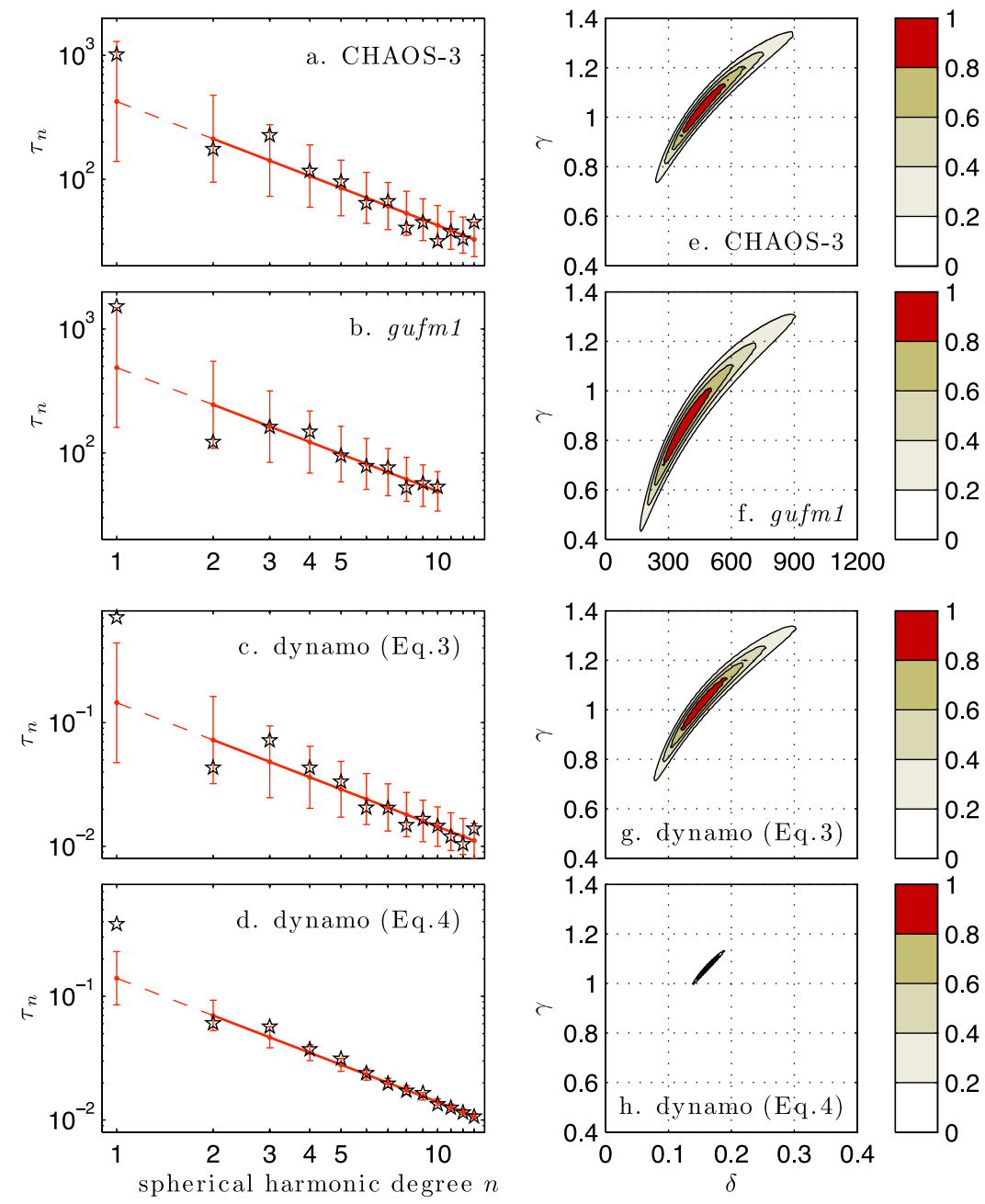

Figure 1. Correlation times $\tau_{n}^{\text {obs }}$ (black stars) and corresponding fits (in red) computed for: (a) the epoch 2005 of CHAOS-3 (using equation (3)); (b) the period 1840-1990 of gufml (using equation (4)); (c) some arbitrary epoch in our dynamo run (using equation (3)); (d) a period of $10 \cdot \tau_{\mathrm{SV}}$ in our dynamo run (using equation (4)). The fits are presented here with a $90 \%$ statistical dispersion bar deduced from the $F^{(2 n+1) N_{n}},(2 n+1) N_{n}-$ pdf for $\left(\tau_{n} / \bar{\tau}_{n}\right)^{2}$, where $N_{n}=1$ for Figures $1 \mathrm{a}-1 \mathrm{c}$. (e-h) The probability density of the two parameters $(\delta, \gamma)$ (normalised by its maximum) for the four cases described in Figures $1 \mathrm{a}-1 \mathrm{~d}$. 


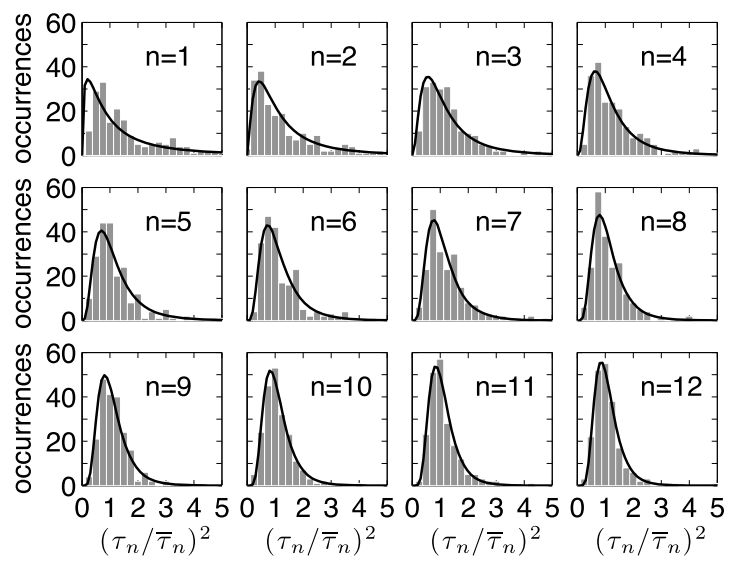

Figure 2. Empirical pdfs of $\left(\tau_{n} / \bar{\tau}_{n}\right)^{2}$ for 200 independent $\tau_{n}$-values of our dynamo run (gray bars). The expected $F^{2 n+1,2 n+1}$-pdfs (black lines) are also shown to check the compliance with the SIS model.

These results are in agreement with the compatibility of an inverse linear law with the data demonstrated in the previous paragraph.

\section{Secular-Variation Timescale in Numerical Dynamo Models}

[13] Using the code PARODY-JA described by Aubert et al. [2008], we now confine our attention to a numerical dynamo driven by compositional convection, which is characterised by the four control parameters $E=10^{-3}, R a^{*}=5$, $P r=1, P m=10$ (following the conventions of Christensen and Aubert [2006]) and operates with a lateral resolution of 64 spherical harmonic degrees. With a magnetic Ekman number $E_{\eta}=10^{-4}$ and a magnetic Reynolds number $R m=$ 232, our dynamo model is located in the "Earth-like" zone of Christensen et al. [2010, Figure 7]. We use this dynamo for illustrative purposes but checked that the results are also applicable to other dynamo solutions, provided that $R m \gtrsim$ 100. In the results discussed in this section, time is expressed in units of the viscous diffusion time.

[14] For comparison with results obtained with geomagnetic field models, we first consider sets of instantaneous correlation times $\tau_{n}$ (computed from equation (3)), and carry out fits for $n=2-13$. Fitting parameters are reported in Table 1 for one typical set of instantaneous values of $\tau_{n}^{\text {obs }}$, and Figure 1c shows the corresponding plot. Figure 1c shows that for $n=2-13$, each $\tau_{n}^{\text {obs }}$ falls within the $90 \%$ statistical dispersion bars, confirming that a one-parameter law of the form $\tau_{\mathrm{SV}} / n$ is also compatible with this simulated field. Note in addition that $\tau_{1}^{\text {obs }}$ now clearly falls out of the statistical dispersion bars.

[15] Since this simulation was integrated for the equivalent of $1000 \cdot \tau_{\mathrm{SV}}$ (based on the $\tau_{\mathrm{SV}}$ we just estimated), it also makes it possible to check that instantaneous values of $\tau_{n}$ fluctuate as predicted by the SIS model. To this end, we compute $\tau_{n}$ from equation (3) every $\Delta t=5 \cdot \tau_{\mathrm{SV}}$ (to conservatively ensure the statistical independence of the resulting 200 values of $\tau_{n}$ ), and estimate $\bar{\tau}_{n}$ from equation (4) using spectra averaged over the entire duration of the run. Figure 2 shows the empirical pdfs of $\left(\tau_{n} \bar{\tau}_{n}\right)^{2}$ for comparison with the expected $F^{2 n+1,2 n+1}$-pdfs. Figure 2 confirms the rel- evance of the SIS model for this study, at the noticeable and expected exception of the dipole field, whose non-zero averaged value violates the SIS assumption and leads to a biased distribution of the values of $\tau_{1}$.

[16] Fits based on $\tau_{n}^{\text {obs }}$ computed for a period $T \approx 10 \cdot \tau_{\mathrm{SV}}$ with the help of equation (4) are also of interest, since they make it possible to check the robustness of our approach when long-term averaged, instead of instantaneous, spectra are considered. Fitting parameters are reported in Table 1 for one such typical set of $\tau_{n}^{\text {obs }}$, and Figure 1d presents the corresponding plot. Figure 1d shows that for each degree $n$, $\tau_{n}^{\text {obs }}$ falls within or very close to the $90 \%$ statistical dispersion bars, which are smaller than previously, due to the impact of time averaging on the number of degrees of freedom (recall Section 2). Interestingly, we also note that $\tau_{\mathrm{SV}}$ is estimated with a much smaller confidence interval, entirely included within the confidence interval previously recovered from instantaneous spectra.

[17] Figures $1 \mathrm{~g}$ and $1 \mathrm{~h}$ show the two-dimensional pdfs (normalised by their maximum, and still denoted by $f$ ) obtained for a two-parameter power law (using equation (7)), for instantaneous and time-averaged spectra, respectively. In the instantaneous case (Figure 1g), and as found for geomagnetic field models, the $\gamma=1$ axis intersects the $f>0.8$ surface, confirming the lack of need to resort to $\bar{a}$ twoparameter power law to account for the observations. The time-averaged case (Figure $1 \mathrm{~h}$ ) is more subtle as it highlights the impact of relying on time-averaged spectra, which require $f(\delta, \gamma)$ to be computed differently (recall Section 2 ). In that case, the $\gamma=1$ axis no longer intersects the $f>0.8$ surface, but the $f>0.2$ surface. This still validates the relevance of the inverse linear law for all practical purposes, while suggesting that more stringent tests could potentially reveal the limits of either the inverse linear law or the SIS model.

[18] In summary, the dynamo simulation indicates that the inverse linear law is a robust candidate to express the dependence of $\tau_{n}$ on $n$, and that instantaneous and timeaveraged spectra statistically lead to the same estimate of the associated secular-variation timescale. In addition, our results show that it is of advantage to resort to time-averaged spectra to increase the robustness of the estimate of $\tau_{\mathrm{SV}}$.

\section{Summary and Discussion}

[19] In this study, we confirmed that the correlation times $\tau_{n}$ in the geomagnetic field and in numerical dynamos could be modelled by a one-parameter law of the form $\tau_{n}=\tau_{\mathrm{SV}} / n$ for the non-dipole field [Christensen and Tilgner, 2004], to within the fluctuations predicted by a SIS model [Hulot and Le Mouël, 1994]. In addition, the statistics we obtained for a more general two-parameter law of the form $\tau_{n}=\delta \times n^{-\gamma}$, support that the value $\gamma=1$ is perfectly acceptable. Using recent satellite models such as POMME-6 [Maus et al., 2010], GRIMM-2 [Lesur et al., 2010] and CHAOS-3 [Olsen et al., 2010], we found a value $\tau_{\mathrm{SV}} \approx 415 \pm_{45}^{55} \mathrm{yr}$. Given the agreement found between instantaneous and "time-averaged" estimates of $\tau_{\mathrm{SV}}$ for the numerical dynamo, we would expect the above figure to be appropriate to describe the dynamics of the geomagnetic field on timescales much longer than that of the historical record. To determine a robust estimate of $\tau_{\mathrm{SV}}$ in geomagnetic field models, we used an algorithm based on $F$-distributions to account for the natural fluctua- 
tions expected for the correlation times. When considering time-averaged spectra, this particular choice of distribution is however less crucial, essentially because $F$-distributions with a large number of degrees of freedom are less skewed, and the fluctuations of the various $\tau_{n}$ more confined. The last line of Table 1 shows accordingly that a least-squares estimate of $\tau_{\mathrm{SV}}$ based on time-averaged spectra of the dynamo model coincides with the more sophisticated estimate based on $F$-statistics. For the sake of simplicity, we would therefore recommend to use a least-squares algorithm to recover the value of $\tau_{\mathrm{SV}}$ from time-averaged spectra in dynamo simulations.

[20] An inverse linear law is indicative of a secular variation governed by convective transport, at least at those scales for which we have observations [Holme and Olsen, 2006]. This behaviour is also conveyed by the inverse dependence of $\tau_{\mathrm{SV}}$ on the magnetic Reynolds number [Christensen and Tilgner, 2004]. In the absence of any better, physically motivated, candidate for the value of the exponent gamma of the two-parameter power law, there is no obvious reason not to choose $\gamma=1$.

[21] From a practical point of view, the secular-variation timescale has already been used to estimate the magnetic dissipation time [Christensen and Tilgner, 2004] and the limit of predictability of the Earth's dynamo [Hulot et al., $2010 \mathrm{~b}$. In the broader context of geomagnetic data assimilation [Fournier et al., 2010], the secular-variation timescale could provide a convenient and sensible means to rescale the time axis of dynamo simulations, by multiplying the non-dimensional time of the numerical model by a factor equal to the ratio of the secular-variation timescale of the Earth (expressed in years) to that of the model (dimensionless, and estimated by applying a least-squares algorithm to time-averaged spectra). The advantage of this procedure is twofold: it is based on a quantity we just found to be reasonably well constrained by the observations, and it implies a consistent rescaling on all those scales effectively probed by the observations. From a dynamical standpoint, as the secular-variation timescale is inversely proportional to the magnetic Reynolds number [Christensen and Tilgner, 2004] and also mildly sensitive to the value of the Ekman number (F. Lhuillier et al., Earth's dynamo limit of predictability controlled by magnetic dissipation, submitted to Geophysical Journal International, 2011), this rescaling would amount to adjusting the level of magnetic turbulence of the numerical model to that of the Earth, while mitigating the discrepancy in kinematic viscosities.

[22] Acknowledgments. We thank U.R. Christensen and an anonymous reviewer for their very useful reviews. F.L. also thanks C. Finlay for his constructive comments on a preliminary version of the manuscript. Numerical computations were performed at S-CAPAD, IPGP, France. This is IPGP contribution 3149.

[23] The Editor thanks Ulrich Christensen and an anonymous reviewer.

\section{References}

Aubert, J., J. M. Aurnou, and J. Wicht (2008), The magnetic structure of convection-driven numerical dynamos, Geophys. J. Int., 172(3), 945-956, doi:10.1111/j.1365-246X.2007.03693.x.
Christensen, U. R., and J. Aubert (2006), Scaling properties of convectiondriven dynamos in rotating spherical shells and application to planetary magnetic fields, Geophys. J. Int., 166(1), 97-114, doi:10.1111/j.1365246X.2006.03009.x.

Christensen, U. R., and A. Tilgner (2004), Power requirement of the geodynamo from ohmic losses in numerical and laboratory dynamos, Nature, 429(6988), 169-171, doi:10.1038/nature02508.

Christensen, U. R., J. Aubert, and G. Hulot (2010), Conditions for Earthlike geodynamo models, Earth Planet. Sci. Lett., 296(3-4), 487-496, doi:10.1016/j.epsl.2010.06.009

Finlay, C. C., et al. (2010), International geomagnetic reference field: The eleventh generation, Geophys. J. Int., 183(3), 1216-1230, doi:10.1111/ j.1365-246X.2010.04804.x.

Fournier, A., G. Hulot, D. Jault, W. Kuang, A. Tangborn, N. Gillet, E. Canet, J. Aubert, and F. Lhuillier (2010), An introduction to data assimilation and predictability in geomagnetism, Space Sci. Rev., 155, 247-291, doi:10.1007/s11214-010-9669-4.

Holme, R., and N. Olsen (2006), Core surface flow modelling from highresolution secular variation, Geophys. J. Int., 166(2), 518-528, doi:10.1111/j.1365-246X.2006.03033.x.

Hongre, L., G. Hulot, and A. Khokhlov (1998), An analysis of the geomagnetic field over the past 2000 years, Phys. Earth Planet. Inter., 106(3-4), 311-335, doi:10.1016/S0031-9201(97)00115-5.

Hulot, G., and J.-L. Le Mouël (1994), A statistical approach to the Earth's main magnetic field, Phys. Earth Planet. Inter., 82(3-4), 167-183, doi:10.1016/0031-9201(94)90070-1.

Hulot, G., C. C. Finlay, C. G. Constable, N. Olsen, and M. Mandea (2010a), The magnetic field of planet Earth, Space Sci. Rev., 152, 159-222, doi:10.1007/s11214-010-9644-0.

Hulot, G., F. Lhuillier, and J. Aubert (2010b), Earth's dynamo limit of predictability, Geophys. Res. Lett., 37, L06305, doi:10.1029/2009GL041869.

Jackson, A., A. R. T. Jonkers, and M. R. Walker (2000), Four centuries of geomagnetic secular variation from historical records, Philos. Trans. $R$. Soc. London, A, 358(1768), 957-990.

Khokhlov, A., G. Hulot, and J. Carlut (2001), Towards a self-consistent approach to palaeomagnetic field modelling, Geophys. J. Int., 145(1), 157-171, doi:10.1111/j.1365-246X.2001.01386.x.

Lesur, V., I. Wardinski, M. Rother, and M. Mandea (2008), GRIMM: The GFZ reference internal magnetic model based on vector satellite and observatory data, Geophys. J. Int., 173(2), 382-394, doi:10.1111/ j.1365-246X.2008.03724.x.

Lesur, V., I. Wardinski, M. Hamoudi, and M. Rother (2010), The second generation of the GFZ Reference Internal Magnetic Model: GRIMM-2, Earth Planets Space, 62(765-773), doi:10.5047/eps.2010.07.007.

Lowes, F. J. (1966), Mean-square values on sphere of spherical harmonic vector fields, J. Geophys. Res., 71(8), 2179.

Lowes, F. J. (1974), Spatial power spectrum of the main geomagnetic field, and extrapolation to the core, Geophys. J. R. Astron. Soc., 36(3), 717-730

Mauersberger, P. (1956), Das Mittel der Energiedichte des geomagnetischen Hauptfeldes an der Erdoberfläche und seine säkulare Änderung, Gerlands Beitr. Geophys., 65, 207-215.

Maus, S., C. Manoj, J. Rauberg, I. Michaelis, and H. Lühr (2010), NOAA/ NGDC candidate models for the 11th generation International Geomagnetic Reference Field and the concurrent release of the 6th generation Pomme magnetic model, Earth Planets Space, 62(10), 729-735, doi:10.5047/eps.2010.07.006.

Olsen, N., H. Lühr, T. J. Sabaka, M. Mandea, M. Rother, L. Tøffner-Clausen, and S. Choi (2006), CHAOS-a model of the Earth's magnetic field derived from CHAMP, Ørsted, and SAC-C magnetic satellite data, Geophys. J. Int., 166(1), 67-75, doi:10.1111/j.1365-246X.2006.02959.x.

Olsen, N., M. Mandea, T. J. Sabaka, and L. Tøffner-Clausen (2010), The CHAOS-3 geomagnetic field model and candidates for the 11 th generation IGRF, Earth Planets Space, 62(10), 719-727, doi:10.5047/ eps.2010.07.003.

Stacey, F. (1992), Physics of the Earth, 3rd ed., Brookfield, Brisbane, Qld., Australia.

von Storch, H., and F. Zwiers (2001), Statistical Analysis in Climate Research, Cambridge Univ. Press, New York.

J. Aubert, A. Fournier, G. Hulot, and F. Lhuillier, Institut de Physique du Globe de Paris, Sorbonne Paris Cité, Université Paris Diderot, INSU/ CNRS, UMR 7154, F-75005 Paris, France. (lhuillier@ipgp.fr) 